Volume 70, Number 2, Pages 965-983(2021)

DOI:10.31801/cfsuasmas.772812

ISSN 1303-5991 E-ISSN 2618-6470

Research Article; Received: July 24, 2020; Accepted: May 21, 2021

\title{
THE LOMAX-LINDLEY DISTRIBUTION: PROPERTIES AND APPLICATIONS TO LIFETIME DATA
}

\author{
Bahman TARVIRDIZADE \\ Department of Mathematics, Maku Branch, Islamic Azad University, Maku, IRAN
}

\begin{abstract}
This paper introduces a new three-parameter distribution which is obtained by combining the Lomax and Lindley distributions in a serial system and is called the Lomax-Lindley distribution. The new distribution is quite flexible to model lifetime data. This distribution provides a simple form for hazard rate function which can be increasing, decreasing, bathtub-shaped and unimodal for different choices of the parameter values. Some statistical properties of the Lomax-Lindley distribution such as quantiles, moments, order statistics, Renyi entropy and mean deviations are discussed. The maximum likelihood estimators of its unknown parameters are obtained and the approximate confidence intervals of the parameters are provided. A Monte Carlo simulation study is conducted to investigate the performance of the maximum likelihood estimators and their corresponding confidence intervals. Finally, two real data sets having bathtub-shaped and unimodal hazard rate functions are analyzed and it is shown that the proposed distribution can provide a better fit than other distributions for both lifetime data.
\end{abstract}

\section{INTRODUCTION}

The Lomax (also known as the Pareto Type II) distribution has been introduced by Lomax [16] as a model for lifetime data analysis. The Lomax distribution is a heavy-tailed distribution and it has wide applications in many fields such as business, economics, actuarial modeling, queuing problems, biological sciences, reliability and life testing problems. For more details we refer to Arnold [5].

The cumulative distribution function (cdf) of the Lomax distribution with two parameters $\alpha$ and $\beta$ is given by

$$
F(x)=1-(1+\beta x)^{-\alpha}, \quad x>0, \quad \alpha, \beta>0 .
$$

Keywords. Lomax distribution, Lindley distribution, maximum likelihood estimation, lifetime data analysis.

๑bahtary@gmail.com

(D) 0000-0002-1517-7365

(C)2021 Ankara University Communications Faculty of Sciences University of Ankara Series A1 Mathematics and Statistics 
Another distribution that has been extensively used over the past decades for modeling data in reliability, biology, insurance, finance and lifetime analysis is Lindley distribution. This distribution was introduced by Lindley [15] in the context of fiducial and Bayesian inference. Ghitany et al. [11] showed that the Lindley distribution is more flexible than the exponential distribution for modeling lifetime data. In recent years, Lindley distribution has been considered by several authors. See, for example, Gupta and Singh [12] and Maiti and Mukherjee [17].

The cdf of the Lindley distribution with parameter $\theta$ is given by

$$
F(x)=1-\left(1+\frac{\theta x}{\theta+1}\right) e^{-\theta x}, \quad x>0, \quad \theta>0 .
$$

Although the Lomax and the Lindley distributions are very useful for modeling lifetime data, they have monotone hazard rate functions. The hazard rate function of Lomax distribution is decreasing and that of Lindley distribution is increasing. Therefore, these distributions may not provide a reasonable parametric fit for modeling phenomena with non-monotone hazard rates such as the bathtub-shaped and unimodal hazard rates which are often encountered in practice. In this regard, some researchers have considered modified forms and generalizations of these distributions to provide more flexibility for describing different types of data. For example, McDonald Lomax distribution by Lemonte and Cordeiro [14], Weibull-Lomax distribution by Tahir et al. [23], Burr X exponentiated Lomax distribution by Aboraya [1], new extended generalized lindley distribution by Maya and Irshad [19], odd log-logistic Lindley distribution by Ozel et al. [21], odd log-logistic Marshal-Olkin Lindley distribution by Alizadeh et al. [3] and exponentiated power Lindley power series class of distributions by Alizadeh et al. [2]. Most of these distributions have four or more parameters which cause estimation problems as a consequence of the number of parameters. On the other hand, the hazard rate functions in most of these models have the complex forms and therefore cannot have many applications for lifetime data analysis in practice. Therefore, major motivation of this study is to introduce a new flexible three-parameter distribution based on the Lomax and the Lindley distributions which its hazard rate function is simple and can cover monotone as well as non-monotone hazard rates.

To obtain more flexible models with simple hazard rate functions, a useful technique is combining the hazard rates of two distributions. For example, additive Weibull distribution by Xie and Lai [25], new modified Weibull distribution by Almalki and Yuan [4] and power-exponential hazard rate distribution by Tarvirdizade and Nematollahi [24] are some models introduced by using this technique. The goal of this article is to propose a new three-parameter lifetime distribution called the Lomax-Lindley (L-L) distribution using the combination of the Lomax and the Lindley distributions in a serial system. The new distribution can be used effectively for analyzing lifetime data since it provides a simple hazard function which can cover increasing, decreasing, bathtub-shaped and unimodal hazard rates. Some properties of the L-L distribution including the density and hazard rate functions, 
quantiles, moments, order statistics, Renyi entropy and mean deviations are presented. The method of maximum likelihood estimation (MLE) is used to estimate the unknown parameters of this new class of distributions. The flexibility of the new model is demonstrated by fitting the L-L distribution to two real data sets having bathtub-shaped and unimodal hazard rate functions.

The contents of this paper are organized as follows. In Section 2, we define the L-L distribution and and study the shape of the hazard rate function of this model. In Section 3, we consider some distributional properties of the new model. In Section 4, the MLEs of unknown parameters are discussed and their asymptotic confidence intervals are provided. In Section 5, a Monte Carlo simulation study is conducted. The applications of the L-L distribution are illustrated by analyzing two real data sets in Section 6. Finally, the conclusions of this paper are given in Section 7 .

\section{The L-L Distribution}

We define the cdf of the $\mathrm{L}-\mathrm{L}$ distribution with three parameters $\alpha, \beta$ and $\theta$ as

$$
F(x)=1-(1+\beta x)^{-\alpha}\left(1+\frac{\theta x}{\theta+1}\right) e^{-\theta x}, \quad x>0, \alpha>0, \beta, \theta \geq 0,
$$

where $\beta+\theta>0$. The probability density function (pdf) of this distribution is given by

$$
f(x)=\left(\frac{\theta^{2}(1+x)}{1+\theta}+\frac{\alpha \beta}{1+\beta x}\left(1+\frac{\theta x}{\theta+1}\right)\right) \begin{array}{r}
(1+\beta x)^{-\alpha} e^{-\theta x}, \\
x>0, \alpha>0, \quad \beta, \theta \geq 0 .
\end{array}
$$

Henceforth, we denote a random variable $X$ having pdf (4) by $X \sim \operatorname{L-L}(\alpha, \beta, \theta)$. The L-L model in (4) can be interpreted as the lifetime of a serial system with two components, one following Lomax distribution with parameters $\alpha$ and $\beta$ and another following Lindley distribution with parameter $\theta$, and the system's lifetime is the minimum of the two components. Clearly, the L-L distribution contains Lomax and Lindley distributions as special cases.

The hazard rate function of the L-L distribution takes a simple form as

$$
h(x)=\frac{\alpha \beta}{1+\beta x}+\frac{\theta^{2}(1+x)}{1+\theta(1+x)} .
$$

To derive the shape of $h(x)$, we obtain the first derivative of (5) as

$$
h^{\prime}(x)=-\frac{\alpha \beta^{2}}{(1+\beta x)^{2}}+\frac{\theta^{2}}{[1+\theta(1+x)]^{2}} .
$$

Setting $h^{\prime}(x)=0$, we obtain

$$
x_{0}=\frac{\sqrt{\alpha} \beta(1+\theta)-\theta}{\theta \beta(1-\sqrt{\alpha})} .
$$


Then it can be seen that the hazard rate function has bathtub-shaped property if

$$
\alpha<1, \beta<\frac{1}{\sqrt{\alpha}}, \theta<\frac{\sqrt{\alpha} \beta}{1-\sqrt{\alpha} \beta},
$$

and the hazard rate function is unimodal if

$$
\alpha>1, \beta<\frac{1}{\sqrt{\alpha}}, \theta>\frac{\sqrt{\alpha} \beta}{1-\sqrt{\alpha} \beta} .
$$

For other values of the parameters $\alpha, \beta$ and $\theta$, the hazard rate function can also be increasing or decreasing. Plots of increasing, bathtub-shaped, decreasing and unimodal hazard rate functions of the L-L distribution and the corresponding pdfs for different values of the parameters $\alpha, \beta$ and $\theta$ are displayed in Figures 1 and 2, respectively.
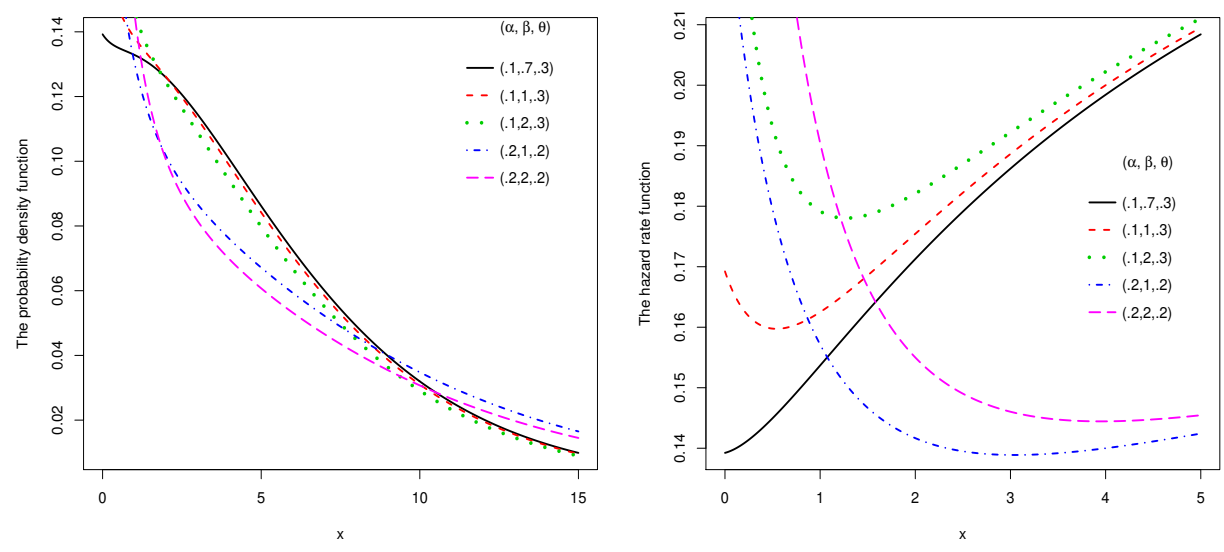

FiguRE 1. The increasing and bathtub-shaped hazard rate functions of the L-L distribution and the corresponding pdfs for different parameter values.

\section{Properties of the L-L Distribution}

We discuss some of the basic statistical properties of the L-L distribution in this section, which consist of quantiles, moments, order statistics, Renyi entropy and mean deviations. 

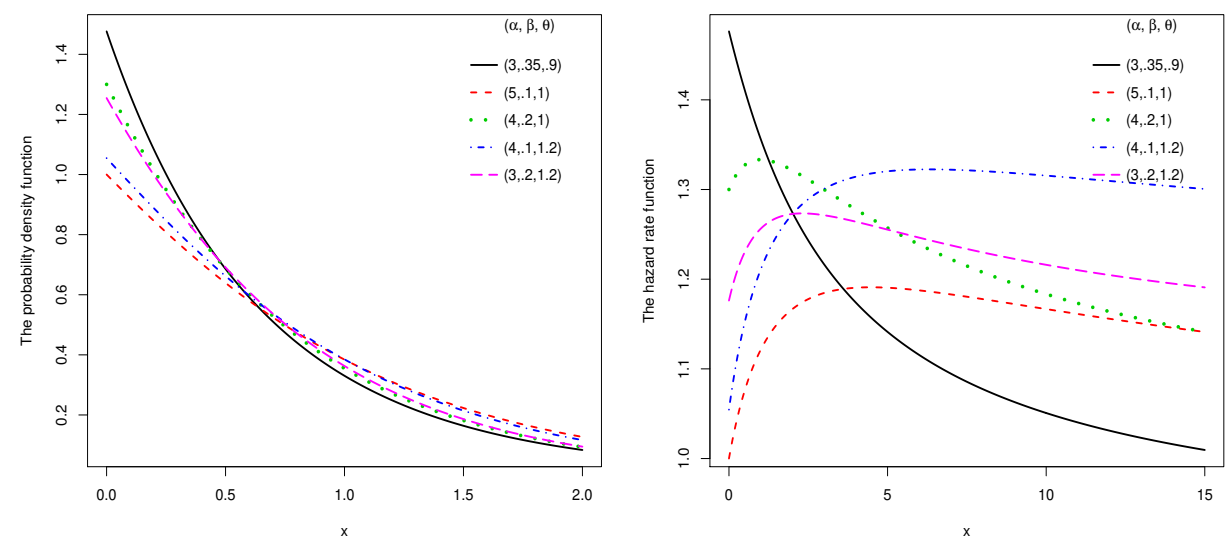

FiguRe 2. The decreasing and unimodal hazard rate functions of the L-L distribution and the corresponding pdfs for different parameter values.

3.1. Quantiles. The quantile function is one of the important functions in probability theory and statistical applications which can be used in data generation from a distribution. The $q$ th quantile $\left(x_{q}\right)$ of the $\mathrm{L}-\mathrm{L}(\alpha, \beta, \theta)$ is obtained by solving $F\left(x_{q}\right)=q$ where $F(x)$ is given in (3). It can be easily shown that $x_{q}$ is the real solution of the following equation

$$
\alpha \log \left(1+\beta x_{q}\right)-\log \left(1+\frac{\theta x_{q}}{\theta+1}\right)+\theta x_{q}+\log (1-q)=0 .
$$

The above equation has no closed form solution in $x_{q}$ and therefore, a numerical technique such as the Newton-Raphson method can be employed to get the quantile. In particular, the median of the $\mathrm{L}-\mathrm{L}(\alpha, \beta, \theta)$ is obtained for $q=0.5$.

3.2. Moments. The $r$ th moment $\left(\mu_{r}\right)$ of the $\mathrm{L}-\mathrm{L}(\alpha, \beta, \theta)$ could be obtained from (4) and integration by parts as follow:

$$
\begin{aligned}
\mu_{r}=E\left(X^{r}\right) & =\int_{0}^{\infty} x^{r}\left(\frac{\theta^{2}(1+x)}{1+\theta}+\frac{\alpha \beta}{1+\beta x}\left(1+\frac{\theta x}{\theta+1}\right)\right)(1+\beta x)^{-\alpha} e^{-\theta x} d x \\
& =\int_{0}^{\infty} r x^{r-1}(1+\beta x)^{-\alpha}\left(1+\frac{\theta x}{\theta+1}\right) e^{-\theta x} d x, \quad r=1,2, \ldots
\end{aligned}
$$

Now, using the Taylor expansion

$$
(1+\beta x)^{-\alpha}=\sum_{i=0}^{\infty}(-1)^{i}\left(\begin{array}{c}
\alpha+i-1 \\
i
\end{array}\right)(\beta x)^{i},
$$


it follows from $(6)$ that

$$
\mu_{r}=\sum_{i=0}^{\infty}(-1)^{i} \beta^{i}\left(\begin{array}{c}
\alpha+i-1 \\
i
\end{array}\right) \int_{0}^{\infty} r x^{r+i-1}\left(1+\frac{\theta x}{\theta+1}\right) e^{-\theta x} d x
$$

Finally, using the transformation $y=\theta x$, we obtain the $r$ th moment of the L$\mathrm{L}(\alpha, \beta, \theta)$ as

$$
\mu_{r}=\sum_{i=0}^{\infty}(-1)^{i} \beta^{i}\left(\begin{array}{c}
\alpha+i-1 \\
i
\end{array}\right) \frac{r}{\theta^{r+i}}\left[\Gamma(r+i)+\frac{1}{\theta+1} \Gamma(r+i+1)\right],
$$

where $\Gamma($.$) is the gamma function.$

Some of the most important characteristics of a distribution can be obtained through moments. For example, the measures of variance, skewness and kurtosis of the L-L distribution can be obtained according to the following relations, respectively,

$$
\sigma^{2}=\mu_{2}-\mu_{1}^{2}, \quad S K=\frac{\mu_{3}-3 \mu_{1} \mu_{2}+2 \mu_{1}^{3}}{\left(\mu_{2}-\mu_{1}^{2}\right)^{3 / 2}}, \quad K U=\frac{\mu_{4}-4 \mu_{1} \mu_{3}+6 \mu_{1}^{2} \mu_{2}-3 \mu_{1}^{4}}{\left(\mu_{2}-\mu_{1}^{2}\right)^{2}} .
$$

3.3. Order statistics. Order statistics make their appearance in many areas of statistical theory and practice. In this subsection, we provide an expression for the pdf of the $i$ th order statistic $\left(X_{i: n}\right)$ of a random sample $X_{1}, X_{2}, \ldots, X_{n}$ drawn from the L-L $(\alpha, \beta, \theta)$. From Balakrishnan and Nagaraja [6], the pdf of $X_{i: n}$ is given by

$$
f_{i: n}(x)=\frac{1}{B(i, n-i+1)} f(x) F(x)^{i-1}(1-F(x))^{n-i}
$$

where $B(.,$.$) is the beta function. Using (3), (4) and the binomial expansion, we$ have

$$
\begin{aligned}
f_{i: n}(x) & =\frac{1}{B(i, n-i+1)} \sum_{j=0}^{i-1}(-1)^{j}\left(\begin{array}{c}
i-1 \\
j
\end{array}\right)\left(\frac{\theta^{2}(1+x)}{1+\theta}+\frac{\alpha \beta}{1+\beta x}\left(1+\frac{\theta x}{\theta+1}\right)\right) \\
& \times\left(1+\frac{\theta x}{\theta+1}\right)^{n-i+j}(1+\beta x)^{-(n-i+j+1) \alpha} e^{-(n-i+j+1) \theta x} \\
& =\frac{1}{B(i, n-i+1)} \sum_{j=0}^{i-1} \sum_{k=0}^{n-i+j}(-1)^{j}\left(\begin{array}{c}
i-1 \\
j
\end{array}\right)\left(\begin{array}{c}
n-i+j \\
k
\end{array}\right)\left(\frac{\theta x}{\theta+1}\right)^{k} \\
& \times\left(\frac{\theta^{2}(1+x)}{1+\theta}+\frac{\alpha \beta}{1+\beta x}\left(1+\frac{\theta x}{\theta+1}\right)\right)(1+\beta x)^{-(n-i+j+1) \alpha} e^{-(n-i+j+1) \theta x} .
\end{aligned}
$$

Some statistical properties of the L-L distribution such as moments, generating function and mean deviations of the order statistics can be derived using (11). 
3.4. Renyi entropy. The entropy is an index for measuring variation or uncertainty of a random variable and has been used in many fields such as physics, engineering and economics among others. A popular entropy measure is Renyi entropy. Renyi entropy of a random variable with pdf $f($.$) is defined as follows:$

$$
I_{R}(r)=\frac{1}{1-r} \log \int_{0}^{\infty} f^{r}(x) d x
$$

where $r>0$ and $r \neq 1$. Let $X \sim \mathrm{L}-\mathrm{L}(\alpha, \beta, \theta)$. Then, using (4) and the binomial expansion, we have

$$
\begin{aligned}
f^{r}(x)= & \sum_{i=0}^{r}\left(\begin{array}{c}
r \\
i
\end{array}\right)(\alpha \beta)^{i}\left(1+\frac{\theta x}{\theta+1}\right)^{i}\left(\frac{\theta^{2}(1+x)}{1+\theta}\right)^{r-i}(1+\beta x)^{-(r \alpha+i)} e^{-r \theta x} \\
= & \sum_{i=0}^{r} \sum_{j=0}^{r-i}\left(\begin{array}{c}
r \\
i
\end{array}\right)\left(\begin{array}{c}
r-i \\
j
\end{array}\right)(-1)^{j}(\alpha \beta)^{i} \theta^{r-i}\left(\frac{1}{\theta+1}\right)^{j}\left(1+\frac{\theta x}{\theta+1}\right)^{r-j} \\
& \times(1+\beta x)^{-(r \alpha+i)} e^{-r \theta x} \\
= & \sum_{i=0}^{r} \sum_{j=0}^{r-i} \sum_{k=0}^{r-j}\left(\begin{array}{c}
r \\
i
\end{array}\right)\left(\begin{array}{c}
r-i \\
j
\end{array}\right)\left(\begin{array}{c}
r-j \\
k
\end{array}\right)(-1)^{j}(\alpha \beta)^{i} \theta^{r-i}\left(\frac{1}{\theta+1}\right)^{j}\left(\frac{\theta x}{\theta+1}\right)^{k} \\
& \times(1+\beta x)^{-(r \alpha+i)} e^{-r \theta x} .
\end{aligned}
$$

Now, using the Taylor expansions of $(1+\beta x)^{-(r \alpha+i)}$, it follows that

$$
\begin{array}{r}
f^{r}(x)=\sum_{i=0}^{r} \sum_{j=0}^{r-i} \sum_{k=0}^{r-j} \sum_{\ell=0}^{\infty}\left(\begin{array}{c}
r \\
i
\end{array}\right)\left(\begin{array}{c}
r-i \\
j
\end{array}\right)\left(\begin{array}{c}
r-j \\
k
\end{array}\right)\left(\begin{array}{c}
r \alpha+i+\ell-1 \\
\ell
\end{array}\right) \\
\times(-1)^{j+\ell}(\alpha \beta)^{i} \theta^{r-i}\left(\frac{1}{\theta+1}\right)^{j}\left(\frac{\theta x}{\theta+1}\right)^{k}(\beta x)^{\ell} e^{-r \theta x}
\end{array}
$$

Finally, by making the transformation $y=r \theta x$ and by identifying a gamma density inside the integral sign, Renyi entropy of $X$ is given by

$$
\begin{aligned}
I_{R}(r)=\frac{1}{1-r} \log & \sum_{i=0}^{r} \sum_{j=0}^{r-i} \sum_{k=0}^{r-j} \sum_{\ell=0}^{\infty}\left(\begin{array}{c}
r \\
i
\end{array}\right)\left(\begin{array}{c}
r-i \\
j
\end{array}\right)\left(\begin{array}{c}
r-j \\
k
\end{array}\right)\left(\begin{array}{c}
r \alpha+i+\ell-1 \\
\ell
\end{array}\right) \\
& \times \frac{(-1)^{j+\ell} \alpha^{i} \beta^{i+\ell} \theta^{r-i+k}}{(\theta+1)^{j+k}} \frac{\Gamma(k+\ell+1)}{(r \theta)^{k+\ell+1}} .
\end{aligned}
$$

The values of some important measures of the L-L distribution such as the median, mean, variance, skewness, kurtosis and Renyi entropy $(r=2)$ for various choices of the parameters $(\alpha, \beta, \theta)$ are presented in Table 1.

3.5. Mean deviations. The totality of deviations from the mean and median is an index for measuring the amount of scatter in a population. Let $X$ be a random 
TABLE 1. The values of some measures of the L-L distribution for different parameter values

\begin{tabular}{ccccccccc}
\hline$\alpha$ & $\beta$ & $\theta$ & Median & Mean & Variance & Skewness & Kurtosis & $I_{R}(2)$ \\
\hline 0.5 & 0.5 & 0.5 & 1.6632 & 2.3438 & 5.1731 & 1.8618 & 8.0781 & 1.5610 \\
0.5 & 0.5 & 3 & 0.2716 & 0.3835 & 0.1392 & 1.8874 & 8.2468 & -0.2493 \\
0.5 & 1 & 0.5 & 1.2725 & 1.9780 & 4.5025 & 2.0830 & 9.2932 & 1.3022 \\
0.5 & 1 & 3 & 0.2512 & 0.3602 & 0.1276 & 1.9563 & 8.6825 & -0.3232 \\
1 & 0.5 & 0.5 & 1.1298 & 1.7308 & 3.4137 & 2.1972 & 10.267 & 1.1925 \\
1 & 0.5 & 3 & 0.2490 & 0.3547 & 0.1219 & 1.9365 & 8.5762 & -0.3338 \\
1 & 1 & 0.5 & 0.7273 & 1.2819 & 2.4594 & 2.6654 & 13.755 & 0.7655 \\
1 & 1 & 3 & 0.2159 & 0.3155 & 0.1027 & 2.0620 & 9.4225 & -0.4701 \\
2 & 0.5 & 0.5 & 0.6514 & 1.0642 & 1.5340 & 2.6978 & 14.694 & 0.6536 \\
2 & 0.5 & 3 & 0.2129 & 0.3074 & 0.0947 & 2.0165 & 9.1454 & -0.4868 \\
2 & 1 & 0.5 & 0.3668 & 0.6666 & 0.7861 & 3.5006 & 23.599 & 0.0906 \\
2 & 1 & 3 & 0.1673 & 0.2500 & 0.0685 & 2.2203 & 10.670 & -0.7202 \\
\hline
\end{tabular}

variable with cdf (3), pdf (4), mean $\mu$ and median $M$. Then, the mean deviation from the mean and the mean deviation from the median are defined by

$$
\delta(\mu)=\int_{0}^{\infty}|x-\mu| f(x) d x=2 \mu F(\mu)-2 I(\mu),
$$

and

$$
\delta(M)=\int_{0}^{\infty}|x-M| f(x) d x=2 M F(M)-M+\mu-2 I(M),
$$

respectively, where

$$
\begin{aligned}
I(a) & =\int_{0}^{a} x f(x) d x=a F(a)-\int_{0}^{a} F(x) d x \\
& =-a(1+\beta a)^{-\alpha}\left(1+\frac{\theta a}{\theta+1}\right) e^{-\theta a}+\int_{0}^{a}(1+\beta x)^{-\alpha}\left(1+\frac{\theta x}{\theta+1}\right) e^{-\theta x} d x,
\end{aligned}
$$

and using 47 and the Taylor expansion of $e^{-\theta x}$,

$$
\begin{aligned}
\int_{0}^{a} & (1+\beta x)^{-\alpha}\left(1+\frac{\theta x}{\theta+1}\right) e^{-\theta x} d x \\
& =\sum_{i=0}^{\infty} \sum_{j=0}^{\infty} \frac{(-1)^{i+j}}{j !}\left(\begin{array}{c}
\alpha+i-1 \\
i
\end{array}\right) \beta^{i} \theta^{j} \int_{0}^{a} x^{i+j}\left(1+\frac{\theta x}{\theta+1}\right) d x \\
& =\sum_{i=0}^{\infty} \sum_{j=0}^{\infty} \frac{(-1)^{i+j}}{j !}\left(\begin{array}{c}
\alpha+i-1 \\
i
\end{array}\right) \beta^{i} \theta^{j}\left(\frac{a^{i+j+1}}{i+j+1}+\frac{\theta}{\theta+1} \frac{a^{i+j+2}}{i+j+2}\right) .
\end{aligned}
$$




\section{MaXimum LiKelihood estimation}

In this section, we apply the maximum likelihood method for estimating the unknown parameters $\alpha, \beta$ and $\theta$. Let $\mathbf{x}=\left(x_{1}, x_{2}, \ldots, x_{n}\right)$ be $n$ observations of a random sample from the $\mathrm{L}-\mathrm{L}(\alpha, \beta, \theta)$. The likelihood function of this sample using (4) can be written as

$L(\alpha, \beta, \theta \mid x)=\prod_{i=1}^{n}\left(\frac{\theta^{2}\left(1+x_{i}\right)\left(1+\beta x_{i}\right)+\alpha \beta\left(1+\theta+\theta x_{i}\right)}{1+\theta}\right)\left(1+\beta x_{i}\right)^{-(\alpha+1)} e^{-\theta x_{i}}$,

and hence the log-likelihood function is given by

$$
\begin{aligned}
\ell(\alpha, \beta, \theta \mid x)= & \sum_{i=1}^{n} \log \left(\theta^{2}\left(1+x_{i}\right)\left(1+\beta x_{i}\right)+\alpha \beta\left(1+\theta+\theta x_{i}\right)\right) \\
& -n \log (\theta+1)-(\alpha+1) \sum_{i=1}^{n} \log \left(1+\beta x_{i}\right)-\theta \sum_{i=1}^{n} x_{i} .
\end{aligned}
$$

Setting the first partial derivatives of $\ell$ with respect to $\alpha, \beta$ and $\theta$ equal to zero, the likelihood equations are obtained in the following form

$$
\begin{gathered}
\frac{\partial \ell}{\partial \alpha}=\sum_{i=1}^{n}\left(\frac{\beta\left(1+\theta+\theta x_{i}\right)}{\theta^{2}\left(1+x_{i}\right)\left(1+\beta x_{i}\right)+\alpha \beta\left(1+\theta+\theta x_{i}\right)}\right)-\sum_{i=1}^{n} \log \left(1+\beta x_{i}\right)=0, \\
\frac{\partial \ell}{\partial \beta}=\sum_{i=1}^{n}\left(\frac{\theta^{2}\left(1+x_{i}\right) x_{i}+\alpha\left(1+\theta+\theta x_{i}\right)}{\theta^{2}\left(1+x_{i}\right)\left(1+\beta x_{i}\right)+\alpha \beta\left(1+\theta+\theta x_{i}\right)}\right)-(\alpha+1) \sum_{i=1}^{n} \frac{x_{i}}{1+\beta x_{i}}=0, \\
\frac{\partial \ell}{\partial \theta}=\sum_{i=1}^{n}\left(\frac{2 \theta\left(1+x_{i}\right)\left(1+\beta x_{i}\right)+\alpha \beta\left(1+x_{i}\right)}{\theta^{2}\left(1+x_{i}\right)\left(1+\beta x_{i}\right)+\alpha \beta\left(1+\theta+\theta x_{i}\right)}\right)-\frac{n}{\theta+1}-\sum_{i=1}^{n} x_{i}=0 .
\end{gathered}
$$

To find the MLEs of $\alpha, \beta$ and $\theta$, say $\hat{\alpha}, \hat{\beta}$ and $\hat{\theta}$, we should solve the above system of non-linear equations (15)-(17) with respect to $\alpha, \beta$ and $\theta$. These equations cannot be solved analytically and therefore, we have to solve the equations numerically. We can use iterative techniques such as a Newton-Raphson type algorithm to obtain the MLEs of the parameters $\alpha, \beta$ and $\theta$. The subroutines to solve non-linear optimization problem are available in $\mathrm{R}$ software. We maximize the log-likelihood function using $n l m()$ package.

To obtain the confidence intervals for the parameters $\alpha, \beta$ and $\theta$, the distributions of the MLEs $\hat{\alpha}, \hat{\beta}$ and $\hat{\theta}$ are needed. Since the MLEs were not obtained in closed forms, then it is not possible to derive their exact distributions. Thus, for interval estimation of the parameters $\alpha, \beta$ and $\theta$, we derive the approximate confidence intervals of the parameters based on the asymptotic distributions of their MLE which is need to calculate the Fisher information matrix. We obtain the observed information matrix since the expected information matrix is very complicated and 
will require numerical integration. The elements of the $3 \times 3$ observed information matrix $I$ are given in the Appendix. Then the asymptotic distribution of the MLE of the parameters $\alpha, \beta$ and $\theta$ is given by

$$
\left(\begin{array}{c}
\hat{\alpha} \\
\hat{\beta} \\
\hat{\theta}
\end{array}\right) \sim N\left(\left(\begin{array}{c}
\alpha \\
\beta \\
\theta
\end{array}\right), \mathbf{V}\right),
$$

where $\mathbf{V}$ is the variance-covariance matrix and can be approximated by the reciprocal of the observed information matrix, i.e., $\mathbf{V}=I^{-1}$. Since $\mathbf{V}$ involves the parameters $\alpha, \beta$ and $\theta$, we replace the parameters by the corresponding MLEs in order to obtain an estimate of $\mathbf{V}$, which is denoted by

$$
\hat{V}=\left(\begin{array}{lll}
\hat{V}_{11} & \hat{V}_{12} & \hat{V}_{13} \\
\hat{V}_{21} & \hat{V}_{22} & \hat{V}_{23} \\
\hat{V}_{31} & \hat{V}_{32} & \hat{V}_{33}
\end{array}\right)=\left(\begin{array}{ccc}
\hat{I}_{11} & \hat{I}_{12} & \hat{I}_{13} \\
\hat{I}_{21} & \hat{I}_{22} & I_{23} \\
\hat{I}_{31} & \hat{I}_{32} & \hat{I}_{33}
\end{array}\right)^{-1},
$$

where $\hat{I}_{i j}$ is the $(i, j)$ th element of the observed information matrix $I$ when $\alpha, \beta$ and $\theta$ are replaced by $\hat{\alpha}, \hat{\beta}$ and $\hat{\theta}$, respectively. Now, using (18), the $100(1-$ $\gamma) \%$ approximate confidence intervals for the parameters $\alpha, \beta$ and $\theta$ are given, respectively, as

$$
\hat{\alpha} \pm z_{\gamma / 2} \sqrt{\hat{V}_{11}}, \quad \hat{\beta} \pm z_{\gamma / 2} \sqrt{\hat{V}_{22}}, \quad \hat{\theta} \pm z_{\gamma / 2} \sqrt{\hat{V}_{33}},
$$

where $z_{\gamma / 2}$ is the $(1-\gamma / 2)$ quantile of the standard normal distribution.

\section{A simulation Study}

In this section, we perform a Monte Carlo simulation study to assess the performance of the point and interval estimates of the parameters presented in Section 4. The performance of the MLEs is compared in terms of their average estimates and mean squared errors (MSEs). We also compare the performance of the confidence intervals in terms of their expected length and coverage probability. To this end, the samples of size $n=10,30,80,150$ are generated from the L-L distribution with three different values for the parameters $(\alpha, \beta, \theta)$, namely, $(0.5,0.5,2),(0.5$, $1,2)$, and $(1.5,0.5,2)$ which correspond to the increasing, bathtub-shaped and unimodal hazard rates, respectively. We report the average estimates and MSEs of the parameters in Table 2. The expected length and coverage probability of the confidence intervals for confidence level $(1-\gamma)=0.95$ are also reported in Table 3 . This simulation study is performed using the statistical software $\mathrm{R}$ and the number of Monte Carlo replications was 5000.

The results of Table 2 indicate that the MSEs for all the selected parameter values decrease with increasing the sample size, which confirm the consistency properties of the MLEs. Based on the results in Table 3, it is observed that increasing the sample size result in a decrease in the expected lengths of the intervals. Also, the assessment of the coverage probabilities show that the approximate confidence 
TABLE 2. Average estimates and MSEs (in parentheses) of the parameters $(\alpha, \beta, \theta)$

\begin{tabular}{cccc}
\hline $\mathrm{n}$ & $\alpha=0.5$ & $\beta=0.5$ & $\theta=2$ \\
\hline 10 & $0.1029(0.4945)$ & $0.0113(0.2918)$ & $2.3134(0.6471)$ \\
30 & $0.0719(0.3537)$ & $0.0134(0.2635)$ & $2.2286(0.2787)$ \\
80 & $0.0058(0.2878)$ & $0.0097(0.2583)$ & $2.2473(0.1249)$ \\
150 & $0.0085(0.2510)$ & $0.0112(0.2488)$ & $2.2568(0.0993)$ \\
\hline $\mathrm{n}$ & $\alpha=0.5$ & $\beta=1$ & $\theta=2$ \\
\hline 10 & $0.0523(0.4019)$ & $0.0584(1.3474)$ & $2.7009(1.3509)$ \\
30 & $0.0382(0.3736)$ & $0.0380(0.9569)$ & $2.4825(0.3880)$ \\
80 & $0.0257(0.3080)$ & $0.0249(0.8908)$ & $2.4428(0.2663)$ \\
150 & $0.0135(0.2483)$ & $0.0139(0.7824)$ & $2.4000(0.1915)$ \\
\hline $\mathrm{n}$ & $\alpha=1.5$ & $\beta=0.5$ & $\theta=2$ \\
\hline 10 & $0.7741(6.5418)$ & $0.1515(0.8265)$ & $2.9206(1.9750)$ \\
30 & $0.2392(2.3196)$ & $0.0669(0.2934)$ & $2.7443(0.7976)$ \\
80 & $0.1907(1.9680)$ & $0.0665(0.2554)$ & $2.6547(0.5210)$ \\
150 & $0.2264(1.8560)$ & $0.0845(0.2158)$ & $2.6232(0.4418)$ \\
\hline
\end{tabular}

TABLE 3. Expected lengths and coverage probabilities (in parentheses) of the parameters $(\alpha, \beta, \theta)$

\begin{tabular}{cccc}
\hline $\mathrm{n}$ & $\alpha=0.5$ & $\beta=0.5$ & $\theta=2$ \\
\hline 10 & $2.3847(0.874)$ & $6.7524(0.951)$ & $3.4349(0.848)$ \\
30 & $1.6174(0.849)$ & $6.3374(0.964)$ & $2.8423(0.890)$ \\
80 & $1.3127(0.870)$ & $5.5297(0.956)$ & $2.3102(0.862)$ \\
150 & $0.9047(0.866)$ & $4.5623(0.978)$ & $1.7505(0.872)$ \\
\hline $\mathrm{n}$ & $\alpha=0.5$ & $\beta=1$ & $\theta=2$ \\
\hline 10 & $2.5894(0.824)$ & $7.7381(0.894)$ & $5.6279(0.944)$ \\
30 & $1.9630(0.806)$ & $6.3784(0.892)$ & $4.1238(0.948)$ \\
80 & $1.1327(0.778)$ & $3.0566(0.898)$ & $2.4911(0.934)$ \\
150 & $0.7995(0.752)$ & $1.9818(0.887)$ & $1.5862(0.942)$ \\
\hline $\mathrm{n}$ & $\alpha=1.5$ & $\beta=0.5$ & $\theta=2$ \\
\hline 10 & $3.6819(0.786)$ & $6.8136(0.978)$ & $5.7652(0.928)$ \\
30 & $2.2182(0.768)$ & $5.4194(0.971)$ & $3.2770(0.904)$ \\
80 & $1.2703(0.757)$ & $3.5746(0.968)$ & $1.8928(0.913)$ \\
150 & $0.8794(0.734)$ & $2.6734(0.973)$ & $1.2594(0.921)$ \\
\hline
\end{tabular}

intervals for most of the parameters provides the coverage probabilities smaller than their nominal level. 


\section{Applications}

In this section, two applications to real data sets with bathtub-shaped and unimodal hazard rates are considered in order to illustrate the potentiality of the L-L distribution. We use goodness-of-fit tests including the Anderson-Darling (AD) and Kolmogorov-Smirnov (K-S) tests to compare the L-L distribution with its sub-models, namely the Lindley and Lomax distributions, and the following threeparameter distributions:

(1) Exponential Lomax (E-Lo) distribution with the pdf

$$
f(x)=\frac{\alpha \lambda}{\beta}\left(\frac{\beta}{x+\beta}\right)^{-\alpha+1} e^{-\lambda\left(\frac{\beta}{x+\beta}\right)^{-\alpha}}, x>0, \alpha, \beta, \lambda>0,
$$

which was introduced by El-Bassiouny et al. [10].

(2) Generalized Lomax (G-Lo) distribution with the pdf

$$
f(x)=\alpha \beta \gamma x^{\gamma-1}\left(1+\beta x^{\gamma}\right)^{-(\alpha+1)}, x>0, \alpha, \beta, \gamma>0,
$$

which was introduced by Maurya et al. [18].

(3) Lindley-Lomax (Li-Lo) distribution with the pdf

$$
f(x)=\frac{\alpha \theta^{2}}{\sigma(\theta+1)}\left[1+\alpha \log \left(1+\frac{x}{\sigma}\right)\right]\left(1+\frac{x}{\sigma}\right)^{-(\alpha \theta+1)}, x>0, \alpha, \theta, \sigma>0,
$$

which was introduced by Cakmakyapan and Ozel [7].

(4) Lindley Weibull (Li-W) distribution with the pdf

$$
f(x)=\frac{\beta \theta^{2}}{(\theta+1)}\left(\alpha^{\beta} x^{\beta-1}+\alpha^{2 \beta} x^{2 \beta-1}\right) e^{-\theta(\alpha x)^{\beta}}, x>0, \alpha, \beta, \theta>0,
$$

which was introduced by Cordeiro et al. [8].

(5) Extended Generalized Lindley (EG-Li) distribution with the pdf

$$
f(x)=\frac{\lambda^{2}(1+x)\left[1-\left(1+\frac{\lambda x}{1+\lambda}\right) e^{-\lambda x}\right]^{\alpha-1}\left\{\alpha+(\gamma-\alpha)\left[1-\left(1+\frac{\lambda x}{1+\lambda}\right) e^{-\lambda x}\right]^{\gamma}\right\}}{(1+\lambda) e^{\lambda x}\left\{\left[1-\left(1+\frac{\lambda x}{1+\lambda}\right) e^{-\lambda x}\right]^{\alpha}+1-\left[1-\left(1+\frac{\lambda x}{1+\lambda}\right) e^{-\lambda x}\right]^{\gamma}\right\}^{2}},
$$

which was introduced by Ranjbar et al. [22].

6.1. Bathtub-shaped hazard rate lifetime data. The first application consists the times between failures (in hours) of load-haul-dump (LHD) machine used to pick up rock or waste. The data has been obtained from Kumar et al. [13] and are presented in Table 4. The TTT-plot presented by Kumar et al. [13] for this data set exhibits a bathtub-shaped hazard rate function. 
TABLE 4. Times between failures of LHD machine (LHD data)

\begin{tabular}{ccccccccccccccccc}
\hline 110 & 13 & 72 & 4 & 45 & 56 & 19 & 27 & 36 & 90 & 19 & 7 & 2 & 118 & 44 & 8 & 277 \\
4 & 8 & 10 & 79 & 103 & 6 & 18 & 147 & 96 & 22 & 3 & 24 & 3 & 9 & 99 & 82 & 121 \\
54 & 79 & 99 & 18 & 5 & 21 & 1 & 3 & 5 & 1 & 59 & 22 & 17 & 35 & 35 & 29 & \\
\hline
\end{tabular}

Table 5 present the MLEs of the model parameters as well as values of A-D statistics, K-S statistics and their corresponding $p$-values for all models. These results show that the L-L distribution has the lowest A-D and K-S values and, has the biggest $p$-value of $\mathrm{K}-\mathrm{S}$ test statistic among all the fitted models. Hence, L-L distribution yields a better fit than Lindley, Lomax, E-Lo, G-Lo, Li-Lo, Li-W and EG-Li distributions under these criteria. Furthermore, Figure 3 show the empirical cdf versus fitted cdfs and the histogram of the data versus fitted pdfs for the LHD data. This figure confirms the goodness-of-fit of L-L distribution with respect to its sub-models and the other competitor distributions.

Substituting the MLE of the unknown parameters in (19), we obtain estimation of the variance-covariance matrix $\hat{V}$ as

$$
\hat{V}=\left(\begin{array}{ccc}
0.1227 & -0.0316 & -0.0017 \\
-0.0316 & 0.0091 & 0.0004 \\
-0.0017 & 0.0004 & 4.4 \times 10^{-5}
\end{array}\right) \text {. }
$$

Therefore, the approximate $95 \%$ confidence intervals of the parameters $\alpha, \beta$ and $\theta$ using (20) are given as $(-0.2207,1.1525),(-0.0966,0.2789)$ and $(0.0077,0.0337)$, respectively.

6.2. Unimodal hazard rate lifetime data. As second application, we consider a clinical trial data set involving survival times (in days) of 45 head and neck cancer patients in arm B which was considered earlier by Efron [9]. The data are presented in Table 6. Mudholkar et al. [20] discussed that this data set indicates a unimodal hazard rate function.

TABle 5. MLEs of parameters, A-D statistic, K-S statistic and corresponding $p$-value

\begin{tabular}{ccccccc}
\hline Distribution & & Estimates & & A-D statistic & K-S statistic & $p$-value \\
\hline $\operatorname{Lindley}(\theta)$ & 0.0432 & - & - & 8.1689 & 0.2383 & 0.0068 \\
$\operatorname{Lomax}(\alpha, \beta)$ & 0.0788 & 7020 & - & 17.006 & 0.5027 & 0.0000 \\
$\operatorname{L-L}(\alpha, \beta, \theta)$ & 0.4658 & 0.0911 & 0.0207 & $\mathbf{0 . 2 8 6 4}$ & $\mathbf{0 . 0 6 9 9}$ & $\mathbf{0 . 9 6 7 2}$ \\
$\operatorname{E-Lo}(\alpha, \beta, \lambda)$ & 0.8621 & 0.0254 & 0.0016 & 0.4053 & 0.0823 & 0.8865 \\
$\operatorname{G-Lo}(\alpha, \beta, \gamma)$ & 77.547 & 0.0005 & 0.8717 & 0.4166 & 0.0830 & 0.8806 \\
$\operatorname{Li}-\operatorname{Lo}(\alpha, \theta, \sigma)$ & 0.3819 & 15.138 & 8203.02 & 0.6425 & 0.0928 & 0.7816 \\
$\operatorname{Li}-\mathrm{W}(\alpha, \beta, \theta)$ & 0.2223 & 0.6868 & 0.3984 & 0.4360 & 0.0845 & 0.8670 \\
$\operatorname{EG}-\operatorname{Li}(\alpha, \gamma, \lambda)$ & 0.4311 & 0.2170 & 0.0198 & 0.4810 & 0.0874 & 0.8386 \\
\hline
\end{tabular}



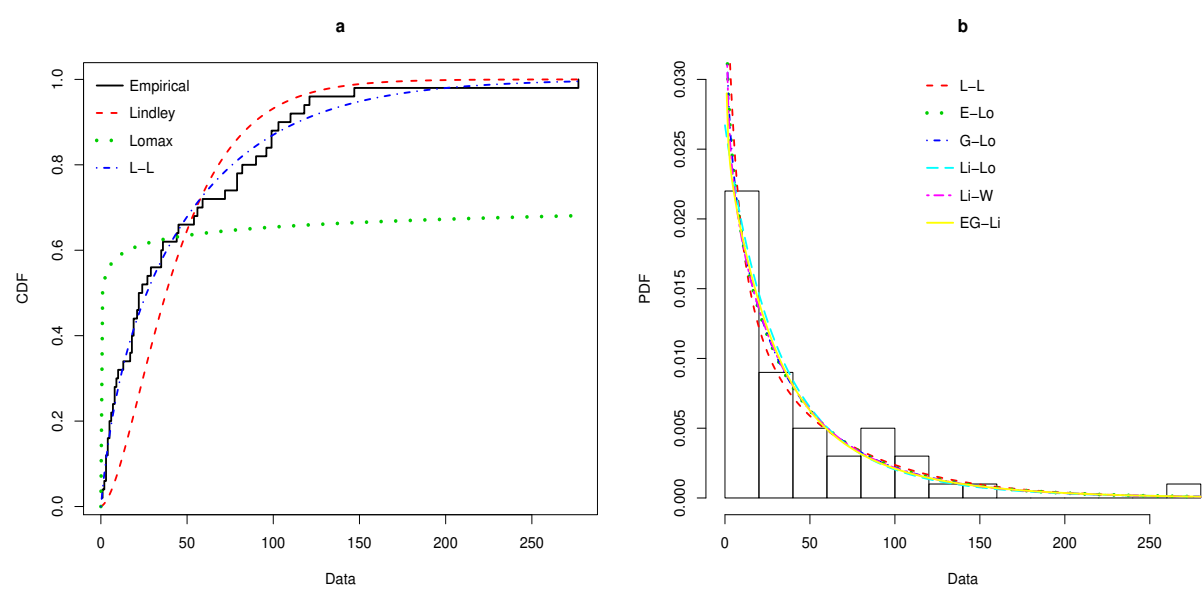

Figure 3. (a) The fitted cdfs and empirical cdf. (b) The fitted pdfs and histogram of the data for LHD data.

TABle 6. Survival times of 45 head and neck cancer patients (Cancer data)

\begin{tabular}{cccccccccccc}
\hline 37 & 84 & 92 & 94 & 110 & 112 & 119 & 127 & 130 & 133 & 140 & 146 \\
155 & 159 & 169 & 173 & 179 & 194 & 195 & 209 & 249 & 281 & 319 & 339 \\
432 & 469 & 519 & 528 & 547 & 613 & 633 & 725 & 759 & 817 & 1092 & 1245 \\
1331 & 1557 & 1642 & 1771 & 1776 & 1897 & 2023 & 2146 & 2297 & & & \\
\hline
\end{tabular}

To compare the goodness of fit of L-L distribution with other distributions, the MLEs of the model parameters as well as values of A-D statistics, K-S statistics and their corresponding $p$-values for all models are calculated and the results are reported in Table 7 . It is observed that the L-L distribution provides the lowest $\mathrm{A}-\mathrm{D}$ and K-S values and, has the biggest $p$-value of K-S test statistic in comparison with its sub-models and the other competitor distributions and therefore, it could be chosen as the best model under these criteria. Figure 4 show the empirical cdf versus fitted cdfs and the histogram of the data versus fitted pdfs for the cancer data. This figure confirms the goodness-of-fit of L-L distribution with respect to all the fitted distributions.

Substituting the MLE of the unknown parameters in (19), we obtain estimation of the variance-covariance matrix $\hat{V}$ as

$$
\hat{V}=\left(\begin{array}{ccc}
0.0937 & 8.6 \times 10^{-5} & -3.1 \times 10^{-5} \\
8.6 \times 10^{-5} & 2.1 \times 10^{-7} & -4.9 \times 10^{-8} \\
-3.1 \times 10^{-5} & -4.9 \times 10^{-8} & 1.8 \times 10^{-7}
\end{array}\right) .
$$


TABLe 7. MLEs of parameters, A-D statistic, K-S statistic and corresponding $p$-value

\begin{tabular}{ccccccc}
\hline Distribution & & Estimates & & A-D statistic & K-S statistic & $p$-value \\
\hline Lindley $(\theta)$ & 0.0031 & - & - & 7.5923 & 0.3037 & 0.0003 \\
Lomax $(\alpha, \beta)$ & 0.0626 & 7266.3 & - & 16.594 & 0.5436 & 0.0000 \\
$\operatorname{L-L}(\alpha, \beta, \theta)$ & 1.5395 & 0.0013 & 0.0010 & $\mathbf{1 . 0 1 8 2}$ & $\mathbf{0 . 1 2 5 8}$ & $\mathbf{0 . 4 3 8 3}$ \\
$\operatorname{E-Lo}(\alpha, \beta, \lambda)$ & 0.9813 & 0.8707 & 0.0015 & 1.3949 & 0.1579 & 0.1904 \\
$\operatorname{G-Lo}(\alpha, \beta, \gamma)$ & 1.1442 & 0.0002 & 1.4413 & 1.0320 & 0.1341 & 0.3608 \\
$\operatorname{Li}-\operatorname{Lo}(\alpha, \theta, \sigma)$ & 146.12 & 0.0151 & 321.13 & 1.0544 & 0.1334 & 0.3663 \\
$\operatorname{Li}-\mathrm{W}(\alpha, \beta, \theta)$ & 0.0001 & 0.9759 & 10.344 & 1.3961 & 0.1582 & 0.1887 \\
$\operatorname{EG}-\operatorname{Li}(\alpha, \gamma, \lambda)$ & 0.5304 & 0.1198 & 0.0012 & 1.2175 & 0.1410 & 0.3027 \\
\hline
\end{tabular}
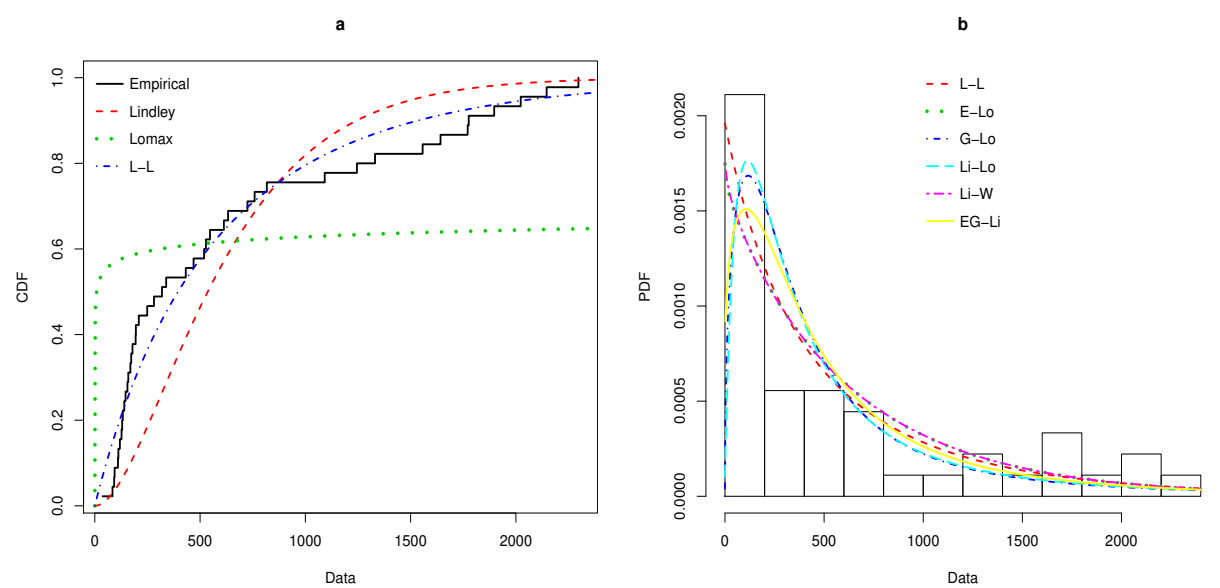

Figure 4. (a) The fitted cdfs and empirical cdf. (b) The fitted pdfs and histogram of the data for cancer data.

Therefore, the approximate $95 \%$ confidence intervals of the parameters $\alpha, \beta$ and $\theta$ using $(20)$ are given as $(0.9395,2.1396),(0.0004,0.0022)$ and $(0.0002,0.0019)$, respectively.

\section{Conclusions}

In this paper, we have proposed a new three-parameter lifetime distribution which is referred to as the the Lomax-Lindley distribution. This distribution is obtained by combining the Lomax and Lindley distributions in a serial system. The new distribution is quite flexible to model lifetime data since it provides a simple form for hazard rate function which can cover increasing, decreasing, bathtubshaped and unimodal hazard rates. We have studied some important mathematical 
properties of new distribution, which consist of quantiles, moments, order statistics, Renyi entropy and mean deviations. The maximum likelihood estimation and asymptotic confidence intervals for the model parameters are also discussed and a simulation study is conducted to evaluate the performances of the point and interval estimates of the parameters. Two real data sets having bathtub-shaped and unimodal hazard rate functions are analyzed to show the superiority of the new distribution. It observed that the present distribution can provide a better fit than other competitor distributions for both lifetime data.

\section{Appendix A}

In Section 4, we used the observed information matrix $I$ to construct the asymptotic confidence intervals for the parameters of the L-L distribution. The elements of this matrix are given by

$$
\begin{aligned}
I_{11} & =\sum_{i=1}^{n}\left(\frac{\beta\left(1+\theta+\theta x_{i}\right)}{g_{i}}\right)^{2}, \\
I_{12} & =-\sum_{i=1}^{n} \frac{\theta^{2}\left(1+x_{i}\right)\left(1+\theta+\theta x_{i}\right)}{g_{i}^{2}}+\sum_{i=1}^{n} \frac{x_{i}}{1+\beta x_{i}}, \\
I_{22} & =\sum_{i=1}^{n}\left(\frac{\theta^{2}\left(1+x_{i}\right) x_{i}+\alpha\left(1+\theta+\theta x_{i}\right)}{g_{i}}\right)^{2}-(\alpha+1) \sum_{i=1}^{n}\left(\frac{x_{i}}{1+\beta x_{i}}\right)^{2}, \\
I_{13} & =\sum_{i=1}^{n} \frac{\beta \theta\left(1+x_{i}\right)\left(1+\beta x_{i}\right)\left(2+\theta+\theta x_{i}\right)}{g_{i}^{2}}, \\
I_{23} & =-\sum_{i=1}^{n} \frac{\alpha \theta^{2}\left(1+x_{i}\right)^{2}-2 \alpha \theta\left(1+x_{i}\right)\left(1+\theta+\theta x_{i}\right)}{g_{i}^{2}}, \\
I_{33} & =-\sum_{i=1}^{n} \frac{2\left(1+x_{i}\right)\left(1+\beta x_{i}\right) g_{i}-\left[2 \theta\left(1+x_{i}\right)\left(1+\beta x_{i}\right)+\alpha \beta\left(1+x_{i}\right)\right]^{2}}{g_{i}^{2}}-\frac{n}{(\theta+1)^{2}},
\end{aligned}
$$

where

$$
g_{i}(\alpha, \beta, \theta)=\theta^{2}\left(1+x_{i}\right)\left(1+\beta x_{i}\right)+\alpha \beta\left(1+\theta+\theta x_{i}\right)
$$

Appendix B

Some programs developed in $\mathrm{R}$ for the L-L distribution fitting and estimation of its parameters are given as follows:

library(MASS)

\# LHD data

$\mathrm{x}=\mathrm{c}(110,13,72,4,45,56,19,27,36,90,19,7,2,118,44,8,277,4,8,10,79$, $103,6,18,147,96,22,3,24,3,9,99,82,121,54,79,99,18,5,21,1,3,5,1,59$, $22,17,35,35,29)$

$\mathrm{n}=50$ 


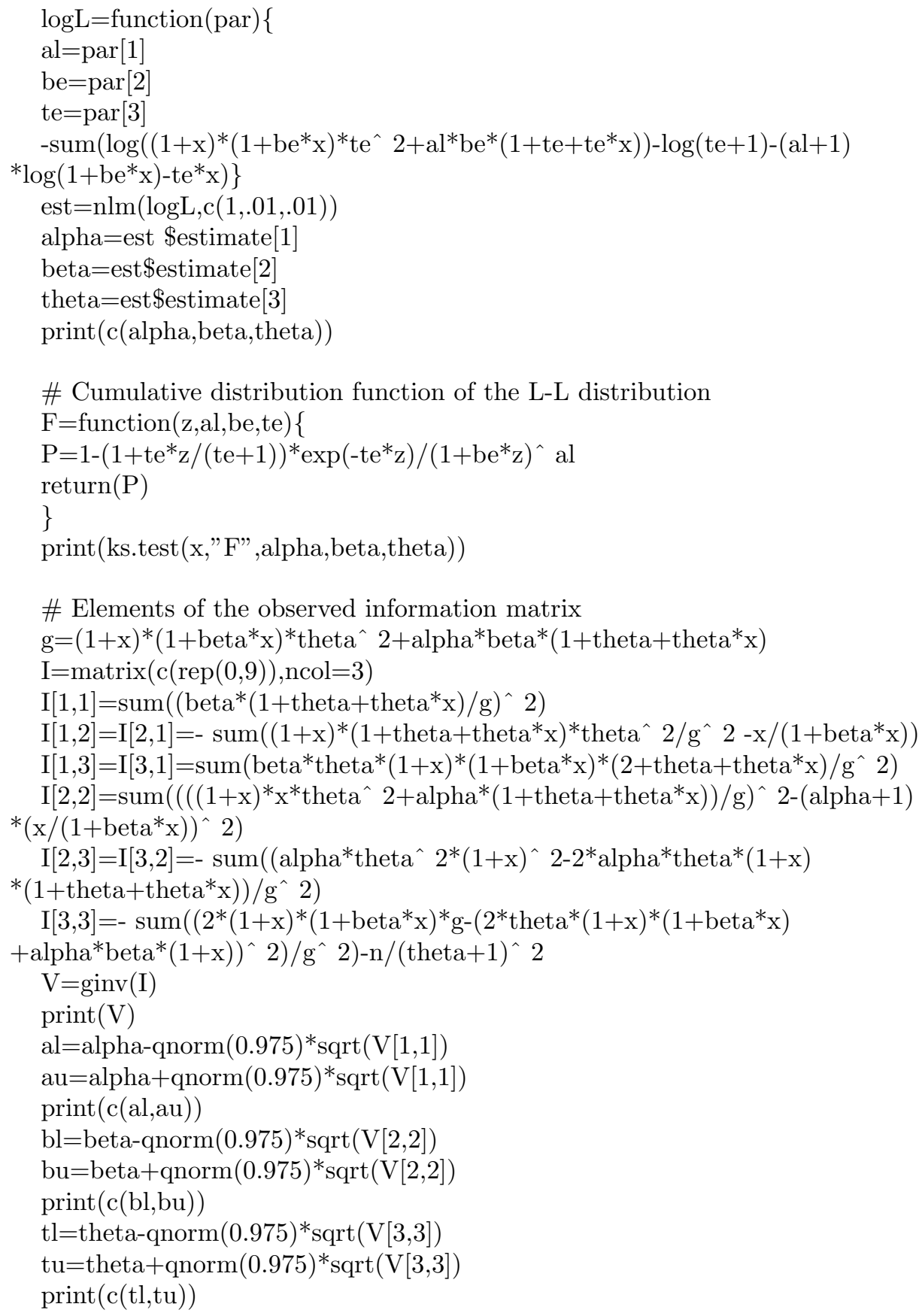


Declaration of Competing Interests The author declares that he has no conflict of interest.

\section{REFERENCES}

[1] Aboraya, M., A new extension of the Lomax distribution with properties and applications to failure times data, Pakistan Journal of Statistics and Operation Research, 15 (2019), 461-479. https://doi.org/10.18187/pjsor.v15i2.2657

[2] Alizadeh, M., Bagheri, S. F., Bahrami Samani, E., Ghobadi, S., Nadarajah, S., Exponentiated power Lindley power series class of distributions: Theory and applications, Communications in Statistics: Simulation and Computation, 47 (2018), 2499-2531. https://doi.org/10.1080/03610918.2017.1350270

[3] Alizadeh, M., Ozel, G., Altun, E., Abdi, M., Hamedani, G. G., The odd log-logistic MarshallOlkin Lindley model for lifetime data, Journal of Statistical Theory and Applications, 16 (2017), 382-400. https://doi.org/10.2991/jsta.2017.16.3.10

[4] Almalki, S. J., Yuan, J., A new modified Weibull distribution, Reliability Engineering and System Safety, 111 (2013), 164-170. https://doi.org/10.1016/j.ress.2012.10.018

[5] Arnold, B. C., Pareto Distributions, International Cooperative Publishing House, Fairland, Maryland, 1983.

[6] Balakrishnan, A. N., Nagaraja, H. N., A First Course in Order Statistics, Wiley-Interscience, New York, 1992. https://doi.org/10.1137/1.9780898719062

[7] Cakmakyapan S., Ozel, G., The Lindley family of distributions: properties and applications, Hacettepe Journal of Mathematics and Statistics, 46 (2017), 1113-1137. https://doi.org/10.15672/HJMS.201611615850

[8] Cordeiro, G. M., Afifi, A. Z., Yousof, H. M., Cakmakyapan, S., Ozel, G., The Lindley Weibull distribution: properties and applications, Anais da Academia Brasileira de Ciências, 90 (2018), 2579-2598. https://doi.org/10.1590/0001-3765201820170635

[9] Efron, B., Logistic regression, survival analysis, and the Kaplan-Meier curve, Journal of the American Statistical Association, 83 (1988), 414-425. https://doi.org/10.1080/01621459.1988.10478612

[10] El-Bassiouny, A. H., Abdo, N. F., Shahen, H. S., Exponential Lomax distribution, International Journal of Computer Applications, 121 (2015), 24-29.

[11] Ghitany, M. E., Atieh, B., Nadarajah, S., Lindley distribution and its application, Mathematics and Computers in Simulation, 78 (2008), 493-506. https://doi.org/10.1016/j.matcom.2007.06.007

[12] Gupta, P. K., Singh, B., Parameter estimation of Lindley distribution with hybrid censored data, International Journal of System Assurance Engineering and Management, 4 (2013), 378-385. https://doi.org/10.1007/s13198-012-0120-y

[13] Kumar, U., Klefsjo, B., Granholm, S., Reliability investigation for a fleet of load haul dump machines in a Swedish mine, Reliability Engineering and System Safety, 26 (1989), 341-361. https://doi.org/10.1016/0951-8320(89)90004-5

[14] Lemonte, A. J., Cordeiro, G. M., An extended Lomax distribution, Statistics, 47 (2013), 800-816. https://doi.org/10.1080/02331888.2011.568119

[15] Lindley, D. V., Fiducial distributions and Bayes theorem, Journal of the Royal Statistical Society, 20 (1958), 102-107. https://doi.org/10.1111/j.2517-6161.1958.tb00278.x

[16] Lomax, K. S., Business failures: another example of the analysis of failure data, Journal of the American Statistical Association, 49 (1954), 847-852. https://doi.org10.1080/01621459.1954.10501239 
[17] Maiti, S. S., Mukherjee, I., On estimation of the PDF and CDF of the Lindley distribution, Communications in Statistics: Simulation and Computation, 47 (2018), 1370-1381. https://doi.org/10.1080/03610918.2017.1311919

[18] Maurya, R. K., Tripathi, Y. M., Lodhi, C., Rastogi, M. K., On a generalized Lomax distribution, International Journal of System Assurance Engineering and Management, 10 (2019), 1091-1104. https://doi.org/10.1007/s13198-019-00839-0

[19] Maya, R., Irshad, M. R., New extended generalized lindley distribution: properties and applications, Statistica, 77 (2017), 33-52. https://doi.org/10.6092/issn.1973-2201/6808

[20] Mudholkar, G. S., Srivastava, D. K., Kollia, G. D., A generalization of the Weibull distribution with application to the analysis of survival data, Journal of the American Statistical Association, 91 (1996), 1575-1583. https://doi.org/10.1080/01621459.1996.10476725

[21] Ozel, G., Alizadeh, M., Cakmakyapan, S., Hamedani, G. G., Ortega, E. M., Cancho, V. G., The odd log-logistic Lindley Poisson model for lifetime data, Communications in Statistics-Simulation and Computation, 46 (2017), 6513-6537. https://doi.org/10.1080/03610918.2016.1206931

[22] Ranjbar, V., Alizadeh, M., Altun, E., Extended Generalized Lindley distribution: properties and applications, Journal of Mathematical Extension, 13 (2019), 117-142.

[23] Tahir, M. H., Cordeiro, G. M., Mansoor, M. and Zubair, M., The Weibull-Lomax distribution: properties and applications, Hacettepe Journal of Mathematics and Statistics, 44 (2015), 455474. https://doi.org/10.15672/HJMS.2014147465

[24] Tarvirdizade, B., Nematollahi, N., A new flexible hazard rate distribution: application and estimation of its parameters, Communications in Statistics: Simulation and Computation, 48 (2019), 882-899. https://doi.org/10.1080/03610918.2017.1402039

[25] Xie, M., Lai, C. D., Reliability analysis using an additive Weibull model with bathtubshaped failure rate function, Reliability Engineering and System Safety, 52 (1995), 87-93. https://doi.org/10.1016/0951-8320(95)00149-2 